\title{
Organ at Risk Doses during High Dose Rate Intracavitary Brachytherapy for Cervical Cancer: A Dosimetric Study
}

\author{
N. V. Vinin*, Joneetha Jones, V. T. Ajas, Geetha Muttath, C. A. Suja, E. K. Nabeel Yahiya, \\ P. N. Shoaib Nawaz, Arun P. Narendran, P. Shimjith \\ Department of Radiation Oncology, Malabar Cancer Centre Thalassery, Kerala, India \\ Email: ^vininnair@gmail.com, joneetha14@gmail.com, ajasvt@gmail.com, geethasatheeshan@gmail.com, sujamitr@gmail.com, \\ nabeelyahiya@gmail.com, shoaibnpn@gmail.com,narunarutmc@gmail.com, shimjith.p.narayanan@gmail.com
}

How to cite this paper: Vinin, N.V., Jones, J., Ajas, V.T., Muttath, G., Suja, C.A., Yahiya, E.K.N., Nawaz, P.N.S., Narendran, A.P. and Shimjith, P. (2018) Organ at Risk Doses during High Dose Rate Intracavitary Brachytherapy for Cervical Cancer: A Dosimetric Study. International Journal of Medical Physics, Clinical Engineering and Radiation Oncology, 7, 472-478.

https://doi.org/10.4236/ijmpcero.2018.74040

Received: July 22, 2018

Accepted: October 9, 2018

Published: October 12, 2018

Copyright $\odot 2018$ by author and Scientific Research Publishing Inc. This work is licensed under the Creative Commons Attribution International License (CC BY 4.0).

http://creativecommons.org/licenses/by/4.0/

\begin{abstract}
Background: Treatment of Cervical cancer includes a combination of external beam radiotherapy (EBRT) with intracavitary brachytherapy (ICBT). ICBT helps to boost radiation dose to primary disease. Organs like rectum, bladder, sigmoid and small bowel lie close to the cervix region and these organs receive dose from EBRT as well as ICBT and we want to know the dose to these organ at risk (OAR). Materials \& Methods: Dosimetric details of 174 ICBT applications done in 58 patients were retrospectively analysed. All patients received EBRT dose of $50.4 \mathrm{~Gy}$ in 28 fractions. All patients had ICBT, three sessions with 7 Gy prescribed to point A. Dosimetric data including dose to right and left point $\mathrm{A}$ and dose to OARs were recorded from Oncentra Planning System. Results: Mean dose to point A on right side was 6.89 Gy and left side was $6.91 \mathrm{~Gy}$. Mean D2cc dose to rectum, bladder, sigmoid and small bowel was 3.5 Gy, 5.25 Gy, 4.75 Gy and 4.2 Gy respectively. Mean EQD2 dose combining EBRT and ICBT in point A was $78.7 \mathrm{~Gy}$ on right side and $79 \mathrm{~Gy}$ on left side. Mean EQD2 doses to D2cc of rectum, bladder, sigmoid and small bowel was 62 Gy, 74.4 Gy, 70.5 Gy and 66.5 Gy respectively. Conclusion: From the results of this dosimetric study it is evident that OARs like rectum, sigmoid, bladder \& bowel are receiving only acceptable doses of radiation using point A prescribed CT based ICBT planning. Hence with regards to OAR doses, CT based ICBT planning with dose prescribed to point $\mathrm{A}$ is a feasible option.
\end{abstract}

\section{Keywords}

Intracavitary Brachytherapy, Carcinoma Cervix, Organ at Risk, High Dose Rate Brachytherapy 


\section{Introduction}

Carcinoma Cervix is the fourth most common cancer among females worldwide. The annual incidence of new cases of cervical cancer worldwide is 528,000. In India, annual incidence of Carcinoma Cervix new cases is around 123,000 [1].

Treatment of locally advanced Carcinoma Cervix has evolved from external beam radiation therapy (EBRT) alone, to EBRT plus brachytherapy, to combined EBRT plus brachytherapy with concurrent chemotherapy [2] [3]. In Carcinoma Cervix radiation treatment, the primary tumour, parametria and pelvic nodes are encompassed in EBRT to a dose to control microscopic disease. Boost to gross disease can be given with the addition of brachytherapy, in turn improving the local control and survival [4] [5] [6] [7] [8].

Image based, three dimensional planning with high dose rate (HDR) treatment delivery is being increasingly used in intracavitary brachytherapy (ICBT) in Carcinoma Cervix. Compared to 3D volume dose prescription and reporting there are limitations for point dose prescription and reporting based on orthogonal radiographic reconstruction of applicators. Studies have shown that $2 \mathrm{D}$ planning gives an incorrect estimation of dose to organ at risk (OAR) [9] [10] [11].

3D image-based planning in cervical cancer brachytherapy has been standardised by the guidelines issued by the Image-Guided Brachytherapy Working Group and the Groupe European de Curethérapie-European Society for Therapeutic Radiology and Oncology (GEC-ESTRO) working group [12] [13].

With this study we aim to find out the dose received by organ at risk (OAR) structures, by intracavitary brachytherapy in Carcinoma Cervix cases treated at our centre.

\section{Methodology}

This retrospective study was done in Department of Radiation Oncology at Malabar Cancer Centre. Data of patients treated with ICBT from ${ }^{\text {st }}$ January 2017 to $31^{\text {st }}$ Dec 2017 were analysed.

\section{1) Inclusion Criteria}

- Carcinoma cervix patients who underwent intracavitary BT with a dose fractionation of $7 \mathrm{~Gy} \times 3$ fractions during the period January 2017 to December 2017.

- EBRT dose was $50.4 \mathrm{~Gy}$ in 28 fractions.

\section{2) Exclusion criteria}

- Intracavitary BT with dose fractionation other than $7 \mathrm{~Gy} \times 3$ fractions, will be excluded from the study.

- EBRT dose other than dosage 50.4 Gy in 28 fractions.

The study was approved by the Institutional Review Board (Ethics committee approval waived of as this is a retrospective study). All patients received external beam radiotherapy (EBRT) with a dose of $50.4 \mathrm{~Gy}$ in 28 fractions. This dosimetric study was completed with 58 patients who underwent HDR-ICBT with Tandem and ovoid/ Ring applicator as a routine treatment from the period 01/01/2017 to 31/12/2017. All patients had three ICBT sessions. Hence dosime- 
tric analysis was done for 174 ICBT applications. Contouring was done on the ONCENTRA Planning System. 7 Gy to point A was prescribed with the standard loading patterns. Various 3D DVH parameters including $0.1 \mathrm{cc}, 1 \mathrm{cc}, 2 \mathrm{cc}$ doses and mean doses to the OARs were noted. Mean dose to right and left point $A$ were noted. EQD2 doses to point A and OARs were noted. These values were noted from ONCENTRA Planning system.

\section{3) Statistical Test}

Basic statistical methods are used such as mean and standard deviation. To compare the mean dose to right and left side point $\mathrm{A}$, $\mathrm{t}$-test was used.

\section{Results}

Dosimetric details of intracavitary brachytherapy (ICBT) for 58 patients were analysed. Each patient had three sessions of ICBT. Hence in the analysis of dosimetric details of 174 intracavitary applications were analysed. Mean dose to right side point A was $6.89 \mathrm{~Gy}$. Mean dose to left side point A was 6.91 Gy. In Table 1 point A doses on right and left side are represented as mean \pm standard deviation.

Using $t$ test mean point $A$ dose on right and left side was compared and showed a p value of 0.54 and hence it was not statistically significant.

As per GEC ESTRO recommendation organ at risk doses are mentioned as D2cc, D1cc, D0.1cc. In our study for rectum mean D2cc, D1cc \& D0.1cc doses were 3.5 Gy, 3.96 Gy \& 5.08 Gy respectively. For Bladder, mean D2cc, D1cc \& D0.1cc doses were $5.25 \mathrm{~Gy}, 5.89$ Gy \& 7.44 Gy respectively. For sigmoid, mean D2cc, D1cc \& D0.1cc doses were 4.75 Gy, 5.41 Gy \& 6.9 Gy respectively. For small bowel, mean D2cc, D1cc \& D 0.1cc doses were 4.2 Gy, 5.56 Gy \& 6.1 Gy respectively. In Table 2, D2cc, D1cc \& D0.1cc doses to organ at risk (OAR) structures like Rectum, bladder, sigmoid \& small bowel are shown as mean \pm standard deviation (Figure 1).

Mean EQD2 dose combining EBRT \& ICBT, to Right \& Left point A are 78.7 Gy \& 79 Gy respectively, assuming $\alpha / \beta$ of 10 for tumor. Assuming a full contribution of dose from EBRT to OARs, the EQD2 to OARs from EBRT \& ICBT was calculated. EQD2 doses to OARs were calculated with $\alpha / \beta$ of 3 . The EQD2 doses to D2cc of rectum, sigmoid, bladder \& small bowel were $62 \mathrm{~Gy}, 70.5 \mathrm{~Gy}, 74.4 \mathrm{~Gy}$ \& 66.5 Gy respectively (Figure 2 ).

Table 1. Showing point A dose on Right and Left side as mean \pm standard deviation.

\begin{tabular}{cc}
\hline Right Point A Dose in Gray (Gy) & Left Point A Dose in Gray \\
\hline $6.89 \pm 0.35$ & $6.91 \pm 0.33$ \\
\hline
\end{tabular}

Table 2. Showing OAR doses as mean \pm standard deviation in Gray (Gy).

\begin{tabular}{ccccc}
\hline & Rectum & Bladder & sigmoid & Bowel \\
\hline D2cc & $3.5 \pm 1.20$ & $5.25 \pm 1.33$ & $4.75 \pm 1.25$ & $4.2 \pm 1.78$ \\
D1cc & $3.96 \pm 1.40$ & $5.89 \pm 1.57$ & $5.41 \pm 1.47$ & $5.56 \pm 1.97$ \\
D0.1cc & $5.08 \pm 1.96$ & $7.44 \pm 2.38$ & $6.9 \pm 2.15$ & $6.1 \pm 2.93$
\end{tabular}




\section{Site wise average dose}

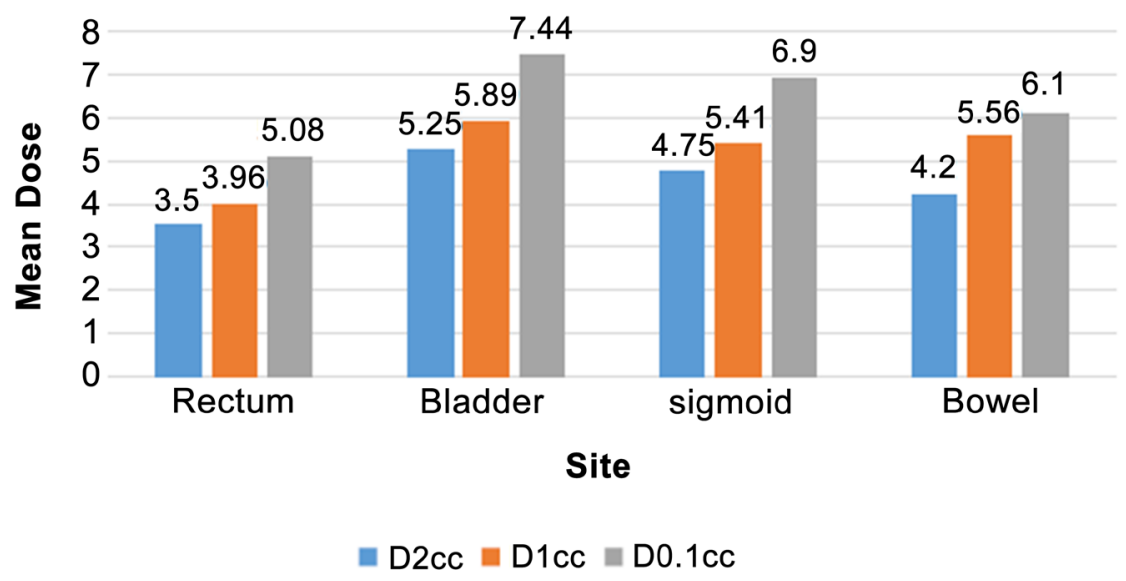

Figure 1. Showing mean dose to OAR structures.

\section{EQD2 doses to D2cc of OARs}

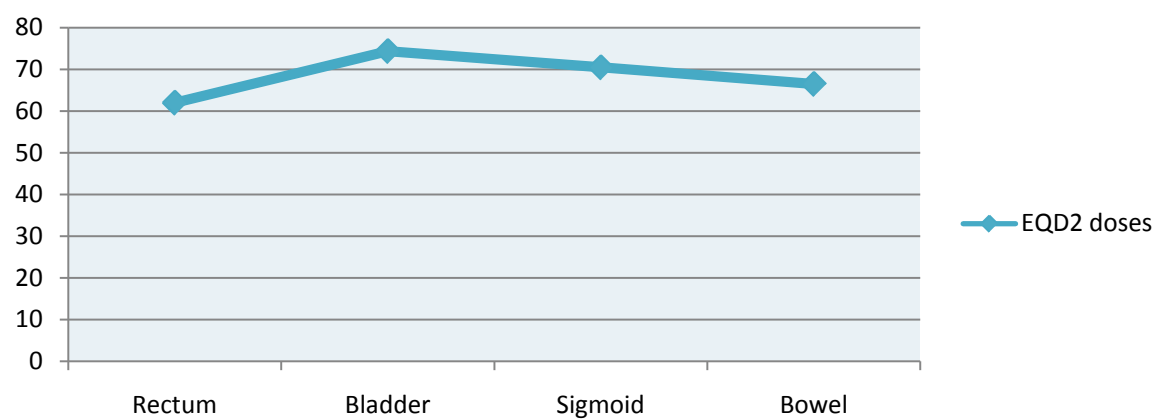

Figure 2. Shows EQD2 doses to D2cc of OARs.

\section{Discussion}

Intracavitary Brachytherapy (ICBT) plays an pivotal role in treatment of Carcinoma Cervix. In our centre CT based planning is done for ICBT. Dose is prescribed to point A. With this retrospective study we wanted to know the doses to adjacent organ at risk structures like rectum, bladder, sigmoid and small bowel. The mean D2cc dose to rectum was $3.5 \mathrm{~Gy}$, that is $50 \%$ of point A prescription dose. Mean D2cc to bladder was 5.25 Gy that is $75 \%$ of point A prescription dose. Mean D2cc to sigmoid was $4.75 \mathrm{~Gy}$, that is $67.86 \%$ of point A prescription dose. Mean D2cc to small bowel was $4.2 \mathrm{~Gy}$, that is $60 \%$ of point A prescription dose. EQD2 doses combining EBRT \& ICBT to OARs are also in acceptable range in this study. Compared to rectum, sigmoid is receiving a slightly higher EQD2 dose. The results of this study denote that all OAR structures are receiving only acceptable level doses.

Bansal et al. in their study documented D2cc dose with ICBT to rectum as $5.38 \mathrm{~Gy}$, to sigmoid as $5.04 \mathrm{~Gy}$ and to bladder as $8.36 \mathrm{~Gy}$ [14]. Compared to that our study showed lower D2cc dose to rectum, sigmoid and bladder. Study done by Arif et al. in which $6 \mathrm{~Gy}$ was the dose prescribed to point A, showed mean 
D2cc dose of $4.58 \mathrm{~Gy}$ to rectum and $4.78 \mathrm{~Gy}$ to bladder [15]. In our study dose prescribed to point A was 7 Gy. Compared to study by Arif et al., mean D2cc dose was less for rectum and slightly higher for bladder.

\section{Conclusion}

From this retrospective dosimetric analysis it is evident that OARs like rectum, sigmoid, bladder \& bowel are receiving only acceptable doses of radiation using point A prescribed CT based ICBT planning. Hence in the new era of image based brachytherapy, point A based ICBT planning is an acceptable option in view of resource constraints with regards to OAR doses. Dosimetric data with regard to OAR doses look promising from our data. However the clinical assessment of individual patients is required to assess the acute and late toxicities associated with ICBT and to know whether the dosimetric data correlate with clinical assessment. On regular follow up history, physical examination and endoscopic evaluation will help us to find out the toxicity profile. Since this was a dosimetric study we have not looked into the clinical profile. We are planning to do future studies to see the correlation between dosimetric data and clinical toxicity profile.

\section{Conflicts of Interest}

The author declares no conflicts of interest regarding the publication of this paper.

\section{References}

[1] GLOBOCAN Cancer Fact Sheets: Cervical Cancer (2018). http://globocan.iarc.fr/old/FactSheets/cancers/cervix-new.asp

[2] Green, J., Kirwan, J., Tierney, J., Symonds, P., et al. (2001) Survival and Recurrence after Concomitant Chemotherapy and Radiotherapy for Cancer of the Uterine Cervix: A Systematic Review and Meta-Analysis. Elsevier. https://www.sciencedirect.com/science/article/pii/S0140673601059657

[3] Recently Updated NCCN Clinical Practice Guidelines in Oncology ${ }^{\mathrm{TM}}$ (2018). https://www.nccn.org/professionals/physician_gls/recently_updated.aspx

[4] Logsdon, M.D. and Eifel, P.J. (1999) FIGO IIIB Squamous Cell Carcinoma of the Cervix: An Analysis of Prognostic Factors Emphasizing the Balance between External Beam and Intracavitary Radiation Therapy. International Journal of Radiation Oncology ${ }^{\star}$ Biology ${ }^{\star}$ Physics, 43, 763-775. https://www.sciencedirect.com/science/article/pii/S0360301698004829 https://doi.org/10.1016/S0360-3016(98)00482-9

[5] Montana, G.S., Martz, K.L. and Hanks, G.E. (1991) Patterns and Sites of Failure In Cervix Cancer Treated in the U.S.A. in 1978. International Journal of Radiation Oncology ${ }^{\star}$ Biology ${ }^{\star}$ Physics, 20, 87-93. http://www.ncbi.nlm.nih.gov/pubmed/1993634 https://doi.org/10.1016/0360-3016(91)90142-Q

[6] Coia, L., Won, M., Lanciano, R., Marcial, V.A., Martz, K. and Hanks, G. (1990) The Patterns of Care Outcome Study for Cancer of the Uterine Cervix Results of the Second National Practice Survey. Cancer, 66, 2451-2456. 
https://doi.org/10.1002/1097-0142(19901215)66:12<2451::AID-CNCR2820661202> 3.0.CO;2-5

[7] Hanks, G.E., Herring, D.F. and Kramer, S. (1983) Patterns of Care Outcome Studies Results of the National Practice in Cancer of the Cervix. Cancer, 51, 959-967. https://doi.org/10.1002/1097-0142(19830301)51:5<959::AID-CNCR2820510533>3.0 .CO;2-K

[8] Lanciano, R.M., Won, M., Coia, L.R. and Hanks, G.E. (1991) Pretreatment and Treatment Factors Associated with Improved Outcome in Squamous Cell Carcinoma of the Uterine Cervix: A Final Report of the 1973 and 1978 Patterns of Care Studies. International Journal of Radiation Oncology ${ }^{\star}$ Biology ${ }^{\star}$ Physics, 20, 667-676. http://www.ncbi.nlm.nih.gov/pubmed/2004942

[9] Jamema, S.V., Saju, S., Mahantshetty, U., Pallad, S., Deshpande, D.D., Shrivastava, S.K., et al. (2008) Dosimetric Evaluation of Rectum and Bladder Using Image-Based CT Planning and Orthogonal Radiographs with ICRU 38 Recommendations in Intracavitary Brachytherapy. Journal of Medical Physics, 33, 3-8.

http://www.ncbi.nlm.nih.gov/pubmed/20041045

https://doi.org/10.4103/0971-6203.39417

[10] Wachter-Gerstner, N., Wachter, S., Reinstadler, E., Fellner, C., Knocke, T.H., Wambersie, A., et al. (2003) Bladder and Rectum Dose Defined from MRI Based Treatment Planning for Cervix Cancer Brachytherapy: Comparison of Dose-Volume Histograms for Organ Contours and Organ Wall, Comparison with ICRU Rectum and Bladder Reference Point. Radiotherapy \& Oncology, 68, 269-276. https://www.sciencedirect.com/science/article/pii/S0167814003001890 https://doi.org/10.1016/S0167-8140(03)00189-0

[11] Fellner, C., Pötter, R., Knocke, T.H. and Wambersie, A. (2001) Comparison of Radiography- and Computed Tomography-Based Treatment Planning in Cervix Cancer in Brachytherapy with Specific Attention to Some Quality Assurance Aspects. Radiotherapy \& Oncology, 58, 53-62.

https://www.sciencedirect.com/science/article/pii/S0167814000002826 https://doi.org/10.1016/S0167-8140(00)00282-6

[12] Mahantshetty, U., Swamidas, J., Khanna, N., Engineer, R., Merchant, N.H. and Shrivastava, S. (2011) Magnetic Resonance Image-Based Dose Volume Parameters and Clinical Outcome with High Dose Rate Brachytherapy in Cervical Cancers-A Validation of GYN GEC-ESTRO Brachytherapy Recommendations. Clinical Oncology, 23, 376-377.

http://linkinghub.elsevier.com/retrieve/pii/S0936655511005590 https://doi.org/10.1016/j.clon.2011.02.006

[13] Nag, S., Cardenes, H., Chang, S., Das, I.J., Erickson, B., Ibbott, G.S., et al. (2004) Proposed Guidelines for Image-Based Intracavitary Brachytherapy for Cervical Carcinoma: Report from Image-Guided Brachytherapy Working Group. International Journal of Radiation Oncology, 60, 1160-1172. https://www.sciencedirect.com/science/article/pii/S0360301604006704 https://doi.org/10.1016/j.ijrobp.2004.04.032

[14] Bansal, A.K., Semwal, M.K., Sharma, D.N., Thulkar, S., Julka, P.K. and Rath, G.K. (2014) A Patient-Based Dosimetric Study of Intracavitary and Interstitial Brachytherapy in Advanced Stage Carcinoma of the Cervix. Journal of Applied Clinical Medical Physics, 15, 4509. http://www.ncbi.nlm.nih.gov/pubmed/24892331 https://doi.org/10.1120/jacmp.v15i3.4509

[15] Ren, J., Menon, G., Sloboda, R., Arif, W.R., Haris, B. and Islamiyah, D.I. (2017) Dose Evaluation of Organs at Risk (OAR) Cervical Cancer Using Dose Volume 
Histogram (DVH) on Brachytherapy Comparative Evaluation of Two Dose Optimization Methods for Image-Guided, Highly-Conformal, Tandem and Ovoids Cervix Brachytherapy Planning Dose Evaluation of Organs at Risk (OAR) Cervical Cancer Using Dose Volume Histogram (DVH) on Brachytherapy. IOP Conf Ser J Phys Conf Ser, 853, 12013.

http://iopscience.iop.org/article/10.1088/1742-6596/853/1/012013/pdf 